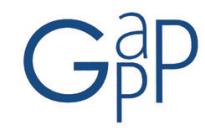

GESTIÓN Y ANÁLISIS DE POLÍTICAS PÚBLICAS, Nueva Época, nº 13 enero-junio 2015 ISSN: 1989-8991

DOI: http://dx.doi.org/10.24965/10.24965/gapp.voi13.10234

\title{
Factores de éxito en la implementación de la E-administración en la educación: el caso de los portales del empleado
}

\author{
Héctor M. Pérez Feijoo \\ Xunta de Galicia \\ Hmpf@edu.xunta.es \\ Mercedes García Ordaz \\ Universidad de Huelva \\ ordaz@uhu.es \\ Francisco José Martínez López \\ Universidad de Huelvo \\ francis@uhu.es
}

\section{Resumen}

La implantación de un portal del empleado en la Administración Pública es un proceso cuyo éxito depende de un conjunto de factores clasificados en cinco áreas críticas: información y datos, tecnología, elementos de la organización, elementos institucionales y, por último, contexto. El presente artículo identifica factores clave en cada categoría para el caso de la implementación de un portal del empleado público educativo. Adicionalmente se establece un orden de prioridades, distinguiendo aquellos factores clave desde el inicio de aquellos a desarrollar en fases posteriores.

Palabras clave

E-Administración, portales del empleado, educación, factores de éxito.

\section{Success factors in the implementation of e-government in the field of education: the case of employee portals}

\section{Abstract}

The implementation of an employee portal in Public Administration is a process whose success depends on a number of factors ranked in five critical areas: information and data, technology, organizational elements, institutional elements and finally context. This article identifies key factors in each category for the implementation of a public education employee portal. Additionally, we establish an order of priorities, distinguishing those key factors from the beginning of those to be developed in later phases.

Keywords

E-Government, employee portals, education, success factors. 


\section{INTRODUCCIÓN}

El propósito de este artículo es realizar un compendio de elementos básicos para la consecución del éxito de la implantación de un portal del empleado en la e-Administración educativa sobre la base de los estudios científicos previos relacionados con esta materia.

El portal del empleado público educativo es la herramienta fundamental para la gestión del conocimiento. La gestión del conocimiento necesita apoyarse en la cultura de la organización para ser eficaz. En el ámbito de la educación la cultura de la organización está indisolublemente vinculada a un conjunto de competencias profesionales de los docentes (Comisión Europea, Dirección General para la Educación y la Cultura, 2005).

El portal del empleado público educativo debe ayudar a la creación de conocimiento basado en estas competencias mediante acciones de e-learning y a la codificación y transferencia del conocimiento útil generado mediante un repositorio de recursos educativos.

A raíz de las necesidades del docente, el portal proporcionará la oferta formativa que le permita alcanzar las competencias de las que carezca o mejorar aquellas otras que requieran de una actualización. En cualquier caso, la adquisición de competencias se basa en la realización de tareas prácticas que midan el rendimiento alcanzado por el docente en el uso de la competencia que se está desarrollando a través de la acción formativa.

La gestión del conocimiento de la organización ha de apoyarse a su vez en un repositorio donde se aloje la información relevante siguiendo un criterio de organización basado en las competencias profesionales. El repositorio permite la recopilación de la información relevante y su reutilización y diseminación a lo largo de toda la organización. Se halla estrechamente relacionado con las actividades formativas ya que como resultado de estas se alimenta el repositorio y a su vez éste puede funcionar como instrumento para la formación.

\section{ESTADO DEL ARTE}

\section{Administración electrónica y portales del empleado}

Según Valenti, Anta y Benderski (2003:69) y Gil-García (2012:12) la administración electrónica, por analogía a las organizaciones privadas, se organiza en cuatro apartados: $\mathrm{G}_{2} \mathrm{C}$-Government to Citizen- que regula las relaciones con los ciudadanos; G2B -Government to Business- encargada de la interacción con las empresas; G2 G -Government to Government- o relaciones con otras Administraciones; y G2E -Government to Employee-, que se ocupa del ámbito del personal al servicio de la Administración. En este contexto, los portales del empleado en la Administración Pública parecen el medio idóneo en el que desarrollar las actividades encuadradas en el G2E.

Los esfuerzos de la Administración pública por modificar el marco de las relaciones de trabajo como una consecuencia lógica de la adaptación de las TIC da como resultado la creación de los llamados portales del empleado público. Un "Portal del Empleado" en una organización de cualquier tipo es definido por Polgar, Bram y Polgar (2006:119) como un punto de acceso único al conjunto de servicios que por vía telemática ofrece esa organización a su personal. En el ámbito empresarial se va generalizando su uso, en particular entre empresas de gran dimensión, pero su aplicación en el sector público se encuentra aún en las primeras fases (Almarabeh y Abuali (2010); Lee, 2010a; Lee, 2010b; Grönlund, 2010; Lee, Chang y Berry, 2011).

\section{Factores de éxito en la e-Administración}

Los estudios que analizan las posibilidades de medición de la eficacia de la e-Administración se clasifican en dos categorías: los que utilizan los resultados directamente relacionados con la implantación del sistema como único instrumento de medida y los que usan fundamentalmente la modificación de las estructuras organizativas como instrumento. Ambas corrientes nos proporcionan una visión a posteriori del grado de consecución de los objetivos iniciales pretendidos al implantar el sistema, pero no nos muestran la causa de este éxito (Abuali, Alawneh y Mohammad, 2010; Gieber, Leitner, Orthofer y Traunmüller, 2010).

Entre los primeros se miden aspectos como la transparencia, la eficacia y la eficiencia o la calidad de los servicios (Bandyopadhyay y Sattarzadeh, 2010; Baqir e lyer 2010; Luna-Reyes et al. 2010; Lux Wigand 2011; Rahman 2010; Smith 2010). Analizando cada uno de estos:

Transparencia y participación activa en la actividad pública: la creación de nuevos canales de comunicación que se deriva de la implantación de la e-Administración hace visibles grandes cantidades de información relevante para el conocimiento de los servicios públicos, mejorando el proceso de rendimiento de cuentas ante el público. Esto no será posible sin el desarrollo de canales de comunicación eficaces y fáciles de usar (Yao, Lee, Hong y Weng., 2010). Por lo tanto, la transparencia, la participación, el grado de apertura y la rendición de cuentas son medidas del éxito de la e-Administración (Pina, Torres y Rollo, 2010) 
Eficiencia y reducción de costes: la mejora en la gestión económica de los servicios públicos, es una de las principales fuentes para la medición del éxito en la implantación de la e-Administración, tanto en lo que respecta a la reducción de los recursos necesarios para su provisión (Bandyopadhyay y Sattarzadeh, 2010; Baqir e lyer., 2010; Rahman, 2010) como en aspectos relacionados con la mejora de la eficiencia (Baqir e lyer., 2010; Corradini, Falcioni, Polini, Polzonetti y Re, 2010). Los estudios empíricos que exploran esta vía encuentran serios problemas para la correcta medición del éxito, como las propias dificultades que existen para la cuantificación de los resultados de la Administración Pública, la falta de una metodología única para la recogida y tratamiento de los datos en diferentes servicios o la indefinición de los objetivos a alcanzar.

Mejoras en la eficacia de las políticas: los objetivos más modestos que se fijan en la implementación inicial de iniciativas de e-Administración como la mejora general en el servicio o la reducción de costes, dejan paso a posteriori a otros más ambiciosos, como la provisión de servicios en áreas cruciales como sanidad o educación (LunaReyes et al. 2010; Olbrich 2010). Es preciso tener en cuenta algunos factores importantes, como que la consecución de resultados en estas áreas no puede ser medida en el corto plazo, que es necesario desarrollar una metodología común para la medición de los resultados obtenidos, y que es necesario preservar la privacidad de información sensible cuando se comparten datos entre varios departamentos para conseguir la medición de la consecución de objetivos (Baqir e lyer, 2010; Carter y McBride 2010).

Mejora de la calidad del servicio: la mayor parte de los estudios sobre este tema la consideran la principal fuente de medición del éxito de las iniciativas relativas a la e-Administración (Baqir e lyer, 2010; Luna-Reyes et al. 2010; Lux Wigand, 2010; Rorissa, Demissie y Pardo, 2010). Las iniciativas para la mejora de los servicios en la Administración siguen los pasos de las desarrolladas en el sector privado para su relación con los clientes. Los principales problemas se encuentran en los problemas de determinados colectivos para el acceso a los servicios online.

FIGURA 1. CONJUNTOS DE INDICADORES DIRECTOS DEL ÉXITO DE LA E-ADMINISTRACIÓN
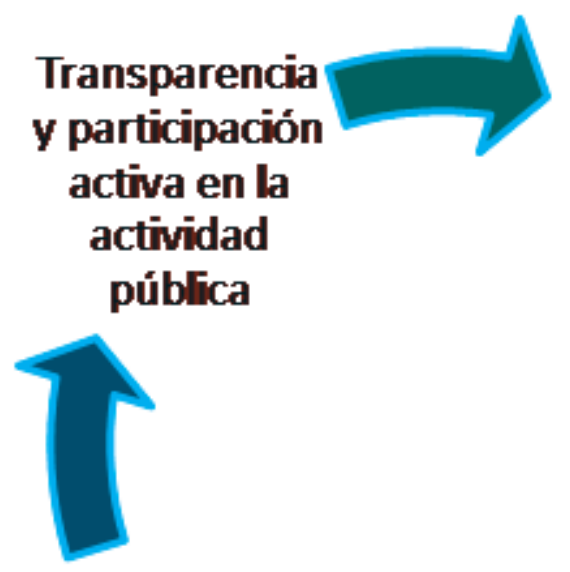

\section{Mejora de la calidad del servicio}

\section{Eficiencia y reducción de costes}

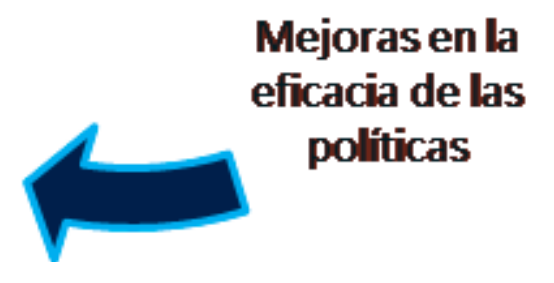

Por lo que respecta a los instrumentos de medida que tienen que ver con las mejoras políticas, organizacionales y técnicas, es evidente que la introducción de tecnologías de la información y las comunicaciones en la Administración pública tiene efectos no solo en los productos o servicios que provee la Administración sino en la propia estructura de las organizaciones públicas y en las percepciones y comportamientos de los quienes se relacionan con ellas (Bandyopadhyay y Sattarzadeh, 2010; Cordella e lannacci 2010). Gil-García (2012:44) afirma que, si bien los resultados de la actuación de las Administraciones Públicas que hemos esbozado en los párrafos anteriores pueden manifestarse o no debido a la gran heterogeneidad de las iniciativas públicas tendentes a implantar la e- 
Administración, la transformación de la estructura organizacional es un factor común a todas ellas, por lo que su estudio se configura como un elemento clave para el análisis del éxito de estas iniciativas (Hanna, 2011). Asimismo, es evidente que la implementación de sistemas basados en tecnologías de la información y las comunicaciones no supone por sí misma un cambio sustancial en la interacción con los usuarios; factores sociales y de la propia organización modulan el éxito de estas iniciativas y deben de ser tenidos en cuenta en una visión amplia. Como hemos enunciado, las mejoras pueden ser de índole organizacional, política o técnica y se caracterizan a grandes rasgos por los siguientes elementos:

- Las mejoras "políticas" hacen referencia a aspectos transversales a la actuación administrativa, como la transparencia, la rendición de cuentas a los ciudadanos, la presentación de información comprensible, la visibilidad de los objetivos de la Administración o la integración de servicios y planes (Ubaldi y Roy, 2010).

- En el ámbito de la estructura organizacional, los cambios implican la necesidad de formación de los empleados, la creación de un conjunto de personas encargadas de asesorar en tecnologías de la información al resto de la organización y la implantación de nuevos canales de comunicación para que puedan realizar su labor de asesoramiento. Entre las mejoras que se obtienen por la modificación de la estructura de la organización se encuentran la reducción de costes, la mejora de los procesos de decisión, la implantación de redes internas amplias, la satisfacción del usuario o la mejora de la coordinación.

- Las mejoras técnicas están relacionadas con la gestión de la información y el procesamiento de datos. Las mejoras se centran, por un lado en el almacenamiento de datos, la eliminación de duplicidades y el tiempo de procesamiento, la ampliación de los estándares de calidad de la información y en la capacidad de compartirla dentro de la propia organización y con otras organizaciones. Por otro lado, en la literatura sobre este tema se atiende en gran cantidad de ocasiones a las mejoras técnicas que afectan a los usuarios del sistema, que son a menudo etiquetadas como accesibilidad, fiabilidad y usabilidad.

FIGURA 2. CONJUNTOS DE INDICADORES RELACIONADOS CON LAS MEJORAS INDIRECTAS DERIVADAS DE LA IMPLANTACIÓN DE LA E-ADMINISTRACIÓN
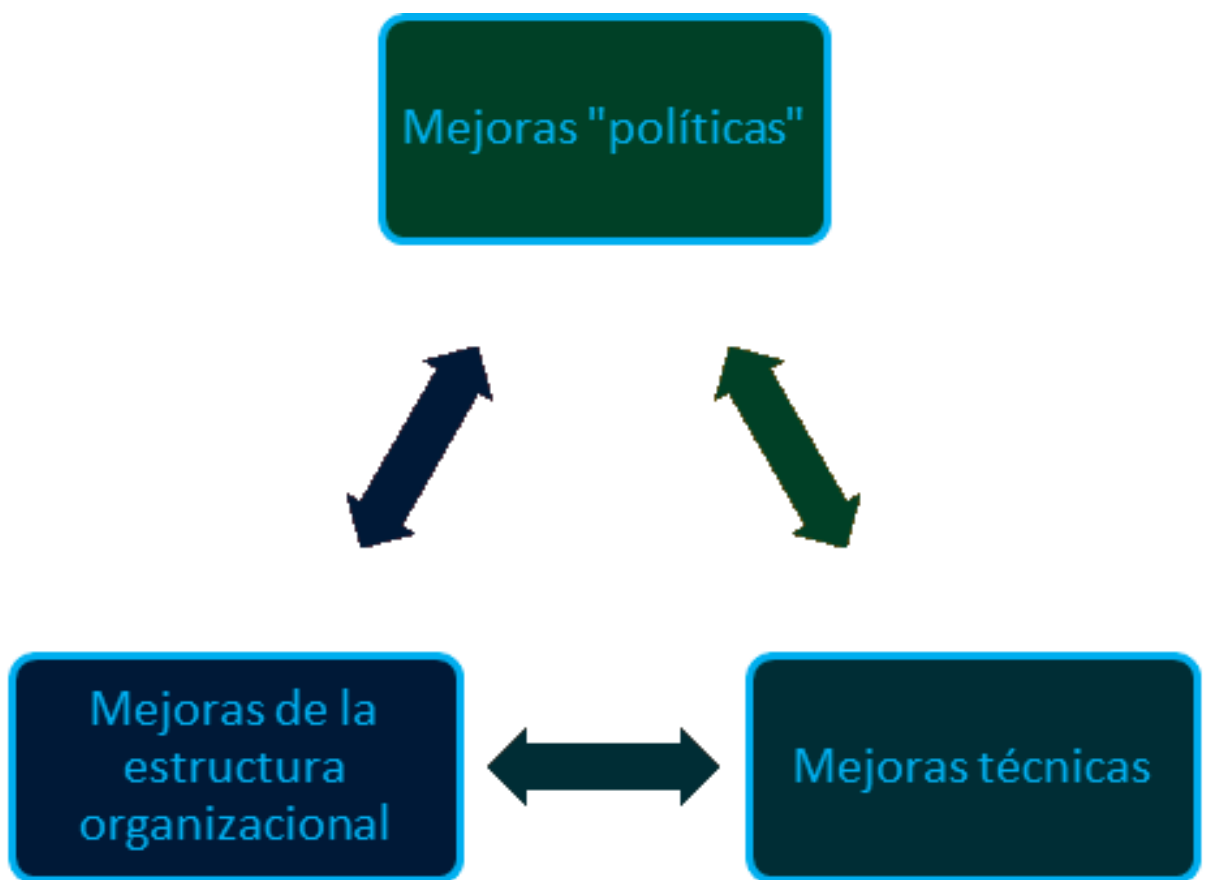

El estudio de los factores que conducen a una implementación exitosa y, por lo tanto, a unos resultados ajustados a los objetivos previstos, ha evolucionado desde varios puntos de vista en la literatura científica. Si bien algunos autores creen que el elemento más significativo es el uso de herramientas administrativas, como la gestión estratégica o la reingeniería (Yu y Jansen, 2010), otros opinan que es el comportamiento individual y la aceptación del cambio el factor determinante (Rowley, 2011). Gil-García (2012:23) sistematiza las distintas corrientes y presenta un esquema con cinco tipos de factores de éxito:

- Factores relacionados con la información y los datos: a pesar de que el mero uso de tecnologías de la información y las comunicaciones supone en múltiples ocasiones la presunción de que la calidad de la información resultante es adecuado, no siempre se obtienen los resultados esperados. La planificación GESTIÓN Y ANÁLISIS DE POLÍTICAS PÚBLICAS, Nueva Época, nº 13 enero-junio 2015 - ISSN: 1989-8991 - DOI: 10.24965/gapp.v0i13.10234 
de los procesos relativos a la gestión de la información es, por lo tanto, necesaria. Los datos pueden ser incompletos, redundantes, innecesarios, contradictorios o extemporáneos; compartir la información puede presentar problemas de compatibilidad en las bases de datos, de información no comparable o de distribución incongruente; o, por último, la distribución de la información es susceptible de verse afectada por falta de soporte político, un marco legal rígido en materia de protección de datos y otros factores político-legales (Yang y Maxwell, 2011).

- Factores relacionados con la tecnología: garantizar la compatibilidad (Baqir e lyer, 2010) o solventar los problemas derivados del uso de tecnologías muy avanzadas y complejas (Choudhari, Banwet y Gupta, 2011) son factores clave para evitar problemas graves en la implantación del sistema. Adicionalmente, el adiestramiento tecnológico del personal de la Administración Pública es un factor de éxito ampliamente reconocido por la literatura científica (Bandyopadhyay y Sattarzadeh, 2010). Aún más difícil es la implantación de un nuevo sistema partiendo de servicios existentes que se pretenden integrar en la nueva estructura. Estos sistemas "heredados" plantean problemas de compatibilidad que en ocasiones no pueden ser solucionados fácilmente (van Veenstra, Klievink y Janssen, 2011, Charalabidis, Lampathaki y Askounis, 2010; Drogkaris, Gritzalis y Lambrinoudakis, 2010).

- Factores organizacionales: procesos, relaciones y estructuras que podemos encontrar dentro de una organización a todos los niveles y que influyen directa o indirectamente en la implantación del sistema. Ejemplos de este tipo de factores son la comprensión de los objetivos de la organización, la amplitud de los cambios planeados, una visión de gestión del proceso, el tamaño del mismo y la diversidad de los usuarios implicados, siendo estos dos últimos de vital importancia (Angelopoulos, Kitsios, Kofakis y Papadopoulos, 2010; Baqir e lyer, 2010; Rorissa et al. 2010; Yang y Maxwell, 2011). Algunos autores sostienen que uno de los principales inconvenientes es la falta de alineación entre los objetivos del proyecto de implantación de la e-Administración y los objetivos generales de la organización (Meijer y Thaens 2010; Valdés et al., 2011), o incluso la existencia de objetivos contradictorios dentro de la organización (LunaReyes et al. 2010). También se señalan como factores determinantes el manejo de las relaciones políticas que se establecen cuando varias Administraciones Públicas conjugan sus esfuerzos para la realización de este tipo de proyectos, o los intereses individuales que llevan a comportamientos desembocan en resistencia al cambio o conflictos internos (Gaudino y Moro, 2010)

- Factores institucionales: leyes, normas en general, costumbres y principios generalmente aceptados en la actuación administrativa. Los factores institucionales son un elemento determinante en la selección, implantación y uso de tecnologías de la información y las comunicaciones en las Administraciones Públicas (Luna-Reyes y Gil-García, 2012). Una enumeración más concreta de estos factores nos indica la existencia de factores políticos y relaciones informales, como las influencias de agentes externos sobre el proceso de toma de decisiones, la influencia de personas con responsabilidad dentro de la propia organización y primacía de proyectos que inciden en conexiones verticales en lugar de vías de cooperación horizontal; o la existencia de preocupaciones individuales acerca de la privacidad y seguridad de la información (Ahn y Bretschneider, 2011; Smith, 2010; Carter et al., 2010; Rose y Grant, 2010; Zhao et al., 2010); y la existencia también de normativa que constriñe la implantación de proyectos de e-Administración, por ejemplo limitaciones en la aplicación temporal de los créditos presupuestarios destinados a los proyectos (Ahn, 2010).

- Factores relacionados con el contexto: no solo es importante estudiar cómo un contexto dado influye sobre la actuación administrativa, sino cómo la propia Administración Pública intenta modificar el entorno en el que se mueve. Los factores relacionados con el contexto son de amplio espectro en la literatura científica: tecnológicos, legales, culturales, políticos, demográficos, económicos y ecológicos.

El conjunto de factores que hemos señalado no debe de ser estudiado únicamente a través de los efectos directos que cada uno de ellos tiene sobre la solución tecnológica que se pretende implementar, sino también prestando atención a las interrelaciones que entre los mismos factores se producen (Gil-García, 2012). En todo caso, el contexto es difícil y, en cualquier caso costoso técnica y económicamente, de controlar.

\section{DISCUSIÓN}

En el caso de la educación superior, nuestra propuesta consiste en la creación de un portal del empleado basado en la gestión del conocimiento. Para ello nos basamos en la integración en los portales del empleado de las competencias profesionales de los docentes. 
La gestión del conocimiento es un factor clave para cualquier organización, pero aún lo es más para la educación, cuyo objetivo es la creación y difusión del mismo. La gestión del conocimiento a través de portales del empleado en Administraciones educativas se centra en la adquisición y mejora de competencias por parte de los profesores (Pérez, 2013) y en la creación y mantenimiento de un repositorio educativo (Vélez-Rivera, RodríguezMartínez, Díaz, Núñez-Molina \& Rivera-Vega, 2010)

Sobre la base de los factores de éxito planteados y prescindiendo del contexto, que como hemos dicho no puede ser controlado con garantías, proponemos los siguientes factores de éxito para un portal del empleado en el ámbito de la educación superior:

- $\quad$ Factores relacionados con información y datos:

- Traspaso correcto de los datos procedentes de las aplicaciones anteriores a la implantación del sistema.

- Medidas para compartir conocimiento con agentes y organizaciones externas.

- $\quad$ Factores relacionados con tecnología:

- C Creación de repositorios educativos estructurados y de uso sencillo.

- Integración de software para la creación de contenidos y entornos virtuales de aprendizaje compatibles con los estándares habituales.

- Personalización de servicios en función de las necesidades de los usuarios.

- Factores organizacionales:

- Determinación de los objetivos a largo plazo en los procesos en los que se comparte conocimiento.

- Establecimiento de las competencias clave y de un plan de formación relacionado con las mismas.

- Identificación de aquellas áreas de la organización en las que la formación del conocimiento se produce con mayor intensidad para establecer una estrategia que potencie su creación.

- $\quad$ Factores institucionales:

- $\quad$ Conocimiento del nivel de competencias clave del profesorado.

- Establecimiento de roles relacionados con la gestión del conocimiento entre el personal docente.

- $\quad$ Medidas para incentivar a los docentes para que compartan conocimiento.

- Reconocimiento de las horas dedicadas a la gestión del conocimiento por parte del personal docente.

- $\quad$ Factores del contexto:

- Formación del profesorado ante las modificaciones de su entorno que implica la implantación del portal.

\section{CONCLUSIONES}

En conclusión, la implantación o mejora de los portales del empleado en el ámbito educativo es una oportunidad para la introducción de un sistema de gestión del conocimiento basado en competencias. En este contexto, el conjunto de medidas planteadas se configura como el grupo de elementos básicos para el éxito de este tipo de iniciativas.

La incidencia de algunos de estos factores de éxito está supeditada a la previa implementación de otros. En el listado que hemos presentado se mencionan primero aquellos factores que son clave para la implementación 
de otros dentro de cada una de las cinco categorías básicas. Así, en el caso de los factores institucionales, es fundamental el conocimiento del nivel de competencias del profesorado para el desarrollo del resto de los factores de éxito. En otros ámbitos, como por ejemplo el tecnológico, la prelación entre los distintos factores de éxito mencionados es difusa, siendo todos ellos de la mayor importancia para el desarrollo de los factores clasificados en otras áreas.

El éxito de un portal del empleado en educación superior es, por lo tanto, un proceso evolutivo donde un núcleo de factores de éxito inicial permite el desarrollo posterior del resto. Entendemos que este conjunto inicial de factores está compuesto por los llamados factores organizacionales (objetivos, competencias clave, estrategias), el traspaso correcto de los datos procedentes de las aplicaciones anteriores a la implantación del sistema, la creación de repositorios educativos estructurados y de uso sencillo, la integración de software para la creación de contenidos y entornos virtuales de aprendizaje compatibles con los estándares habituales, el conocimiento del nivel inicial de competencias clave de cada profesional y la formación del profesorado ante las modificaciones de su entorno que implica la implantación del portal.

\section{CUADRO 1. PRIORIDADES ENTRE LOS FACTORES DE ÉXITO}
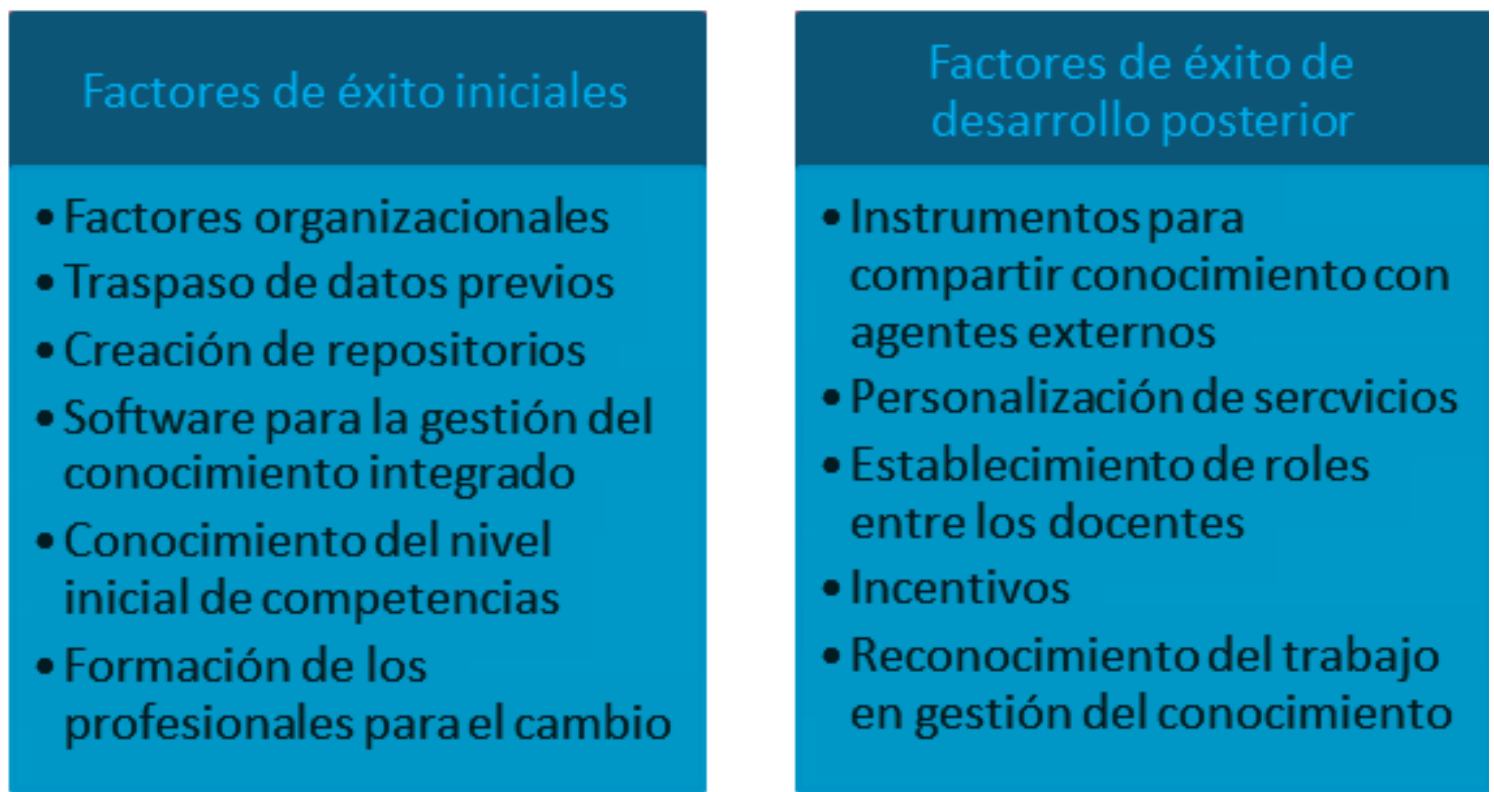

En definitiva, el éxito en la implementación de un portal del empleado en el ámbito de la educación superior implica no solo tener en cuenta un conjunto de factores de éxito, sino también que existen prioridades entre ellos. El portal se basará en un primer momento en un núcleo reducido de funcionalidades basadas en factores de éxito inicial para ampliarse posteriormente sobre la base de un conjunto más amplio de elementos fundamentales.

\section{BIBLIOGRAFÍA}

ABUALI A; ALAWNEH A; y MOHAMMAD H (2010), "Factors and Rules Effecting In E-Government", European Journal of Scientific Research, vol. 39, num. 2.

AHN, M J (2011), “Adoption of e-communication applications in U.S. municipalities: The role of political environment, bureaucratic structure, and the nature of applications", The American Review of Public Administration, vol. 41, pp. 428-452. DOI: 10.1177/0275074010377654.

AHN, M J; BRETSCHNEIDER, S (2011), “Politics of E-Government: E-Government and the Political Control of Bureaucracy”, Public Administration Review, vol. 71, num. 3, pp. 414-424. DOI: 10.1111/j.1540-6210.2011.02225.X

ALMARABEH, T; y ABUALI, A (2010), “A General Framework for E-Government: Definition Maturity Challenges, Opportunities, and Success”, European Journal of Scientific Research, vol.39, num.1, pp.29-42. 
BANDYOPADHYAY, A; y SATTARZADEH, S (2010), "A challenging e-journey along the silk road: lessons learned from e-governments in China and India”, en Reddick CG (ed.): Comparative e-government, pp. 116-138, New York, Springer. DOI: $10.1007 / 978-1-4419-6536-3 \_6$.

BAQIR MN; e IYER L (2010), "E-government maturity over 10 years: a comparative analysis of e-government maturity in select countries around the world", en Reddick CG (ed.): Comparative e-government, pp. 3-22, New York, Springer. DOI: 10.1007/978-1-4419-6536-3_1.

CARTER L; y MCBRIDE A (2010), “Information privacy concerns and e-government: a research agenda”, Transforming Government: People, Process and Policy, vol. 4, num. 1, pp. 10-13. DOI: 10.1108/17506161011028777.

CHARALABIDIS Y; LAMPATHAKI F; y ASKOUNIS D (2010), "Emerging interoperability directions in electronic government", en H. J. Popplewell K (Ed.): Enterprise Interoperability IV: making the internet of the future for the future of enterprise, pp. 419-428, London, Springer. DOI: 10.1007/978-1-84996-257-5_39.

CHOUDHARI RD; BANWET DK; y GUPTA, MP (2011), “Assessment of risk in e-governance projects: An application of product moment correlation and cluster analysis techniques", Electronic Government, an International Journal, vol. 8, num. 1, pp. 85-102. DOI: 10.1504/EG.2011.037699.

COMISIÓN EUROPEA, DIRECCIÓN GENERAL PARA LA EDUCACIÓN Y LA CULTURA (2005), Principios Europeos Comunes sobre las Competencias y Cualificaciones del Profesorado, informe del Directorate-General for Education and Culture.

CORDELLA A; y IANNACCI F (2010), "Information systems in the public sector: The e-Government enactment framework”, The Journal of Strategic Information Systems, vol. 19, num. 1, pp. 52-66. DOI: 10.1016/j.jsis.2010.01.001.

CORRADINI F, POLZONETTI A, RE B, y TESEI L (2008), "Quality of service in e-government underlines the role of information usability”, International Journal of Information Quality, vol. 2, num. 2, pp. 133-151. DOI: 10.1504/ IJIQ.2008.022960.

DROGKARIS P; GRITZALIS S; LAMBRINOUDAKIS C (2010), "Transforming the Greek e-government environment towards the e-gov 2.0 era", en F. E. Andersen KN (Ed.), Procceedings of the 1st international conference, EGOVIS 2010, pp. 142-149, Berlin, Springer. DOI: 10.1007/978-3-642-15172-9_14.

GAUDINO S; MORO G (2010), "Evaluation of an e-government project: which are the barriers to e-government integration?", International Journal of Technology, Policy and Management, vol. 10, num. 1, pp. 53-72. DOI: 10.1504 L IJTPM.2010.032853.

GIL-GARCÍA JR (2012), Enacting Electronic Government Success: An Integrative Study of Government-wide Websites, Organizational Capabilities, and Institutions, New York, Springer. DOI: 10.1007/978-1-4614-2015-6.

GRÖNLUND A (2010), "Ten years of e-government: the "end of history" and new beginning”, Proceedings of the 9th IFIP WG 8.5 international conference EGOV 2010, pp. 13-24, Berlin, Springer. DOI: 10.1007/978-3-642-147999 2.

GIEBER H; LEITNER C; ORTHOFER G; y TRAUNMÜLLER R (2010), “Taking the best practice forward”, en B. L. Chen H-G (ed.): Digital government: e-government research, case studies and implementation, pp. 203-218, New York, Springer. DOI: $10.1007 / 978-0-387-71611-4 \_23$.

HANNA, NK (2011), Transforming government and building the information society: challenges and opportunities for the developing world, Springer, New York. DOI: 10.1007/978-1-4419-1506-1.

LEE J (2010), “10year retrospect on stage models of e-Government: A qualitative meta-synthesis”, Government Information Quarterly, vol. 27, num. 3, pp. 220-230. DOI: 10.1016/j.giq.2009.12.009.

LEE JY (2010), “The development of e-government capabilities: framework for government”, en R H (ed.): Handbook of research on e-government readiness for information and service exchange: utilizing progressive information communication technologies , pp. 1-20, Hershey, IGI Global. DOI: 10.4018/978-1-60566-671-6.choo1.

LEE C-P; CHANG K; y BERRY F S (2011), "Testing the Development and Diffusion of E-Government and E-Democracy: A Global Perspective”, Public Administration Review, vol. 71, num. 3, pp. 444-454. DOI: 10.1111/j.15406210.2011.02228.x. 
LUNA-REYES L F; PARDO T A; GIL-GARCIA J R; NAVARRETE C; ZHANG J; y MELLOULI S (2010), “Digital Government in North America: a comparative analysis of policy and program priorities in Canada, Mexico and the United States", en Reddick CG (ed.): Comparative e-government, pp. 139-160, New York, Springer. DOI: 10.1007/978-1-44196536-3_7.

LUNA-REYES L F; y GIL-GARCÍA J R (2011), “Using institutional theory and dynamic simulation to understand complex e-Government phenomena”, Government Information Quarterly, vol. 28, num. 3, pp. 329-345. DOI: 10.1016/i.giq.2010.08.007.

MEIJER, A; y THAENS M (2010), "Alignment 2.0: Strategic use of new internet technologies in government”, Government Information Quarterly, 2010, vol. 27, num. 2, pp. 113-121. DOI: 10.1016/j.giq.2009.12.001.

OLBRICH S (2010), “Implementing e-government locally - an empirical survey from the European Metropolitan area Rhine-Neckar”, en Reddick CG (ed.): Comparative e-government, pp. 221-237, New York, Springer. DOI: 10.1007/978-1-4419-6536-3_11.

PÉREZ H (2013), Portales del empleado en la e-Administración. Estudio sobre la Administración electrónica educativa española, PhD Thesis, Universidad de Huelva.

PINA, V; TORRES, L; y ROYO, S (2010), "Is e-government leading to more accountable and transparent local governments? An overall view”, Financial Accountability \& Management, vol. 26, num. 1, pp. 3-20. DOI: 10.1111/j.14680408.2009.00488.x.

POLGAR J; BRAM R; y POLGAR T (2006), Building and Managing Enterprise Wide Web. Idea Group.

RAHMAN H (2010), “Framework of e-governance at the local government level”, en Reddick CG (ed.): Comparative e-government, pp. 23-47, New York, Springer. DOI: 10.1145/1693042.1693121.

RORISSA, A; DEMISSIE, D; PARDO, T (2011),"Benchmarking e-government: A comparison of frameworks for computing e-government index and ranking", Government Information Quarterly, vol. 28, num. 3, p.p. 354-362. DOI:10.1016/j.giq.2010.09.006.

ROSE, W R; GRANT, G G (2010), "Critical issues pertaining to the planning and implementation of e-government initiatives", Government Information Quarterly, vol. 27, num. 1, pp. 26-33. DOI: 10.1016/j.giq.2009.06.002.

ROWLEY J (2011), “E-government stakeholders- who are they and what do they want?", International Journal of Information Management , vol. 31, num. 1, pp. 53-62. DOI: 10.1016/j.ijinfomgt.2010.05.005.

SMITH ML (2010), "Building institutional trust through e-government trustworthiness cues", Information Technology \& People, vol. 23, num. 3, pp.222 - 246. DOI: 10.1108/09593841011069149.

UBALDI B-C; y Roy J (2010), "E-Government and Federalism in Italy and Canada-A Comparative Assessment", en Reddick CG (ed.): Comparative e-government, pp. 183-199, New York, Springer. DOI: 10.1007/978-1-4419-65363_9.

VALDÉS, G; SOLAR, M; ASTUDILLO, H; IRIBARREN, M; CONCHA, G; y VISCONTI, M (2011), “Conception, development and implementation of an egovernment maturity model in public agencies", Government Information Quarterly, vol. 28, num. 2, pp. 176-187. DOI: 10.1016/j.giq.2010.04.007.

VALENTI, P; ANTA, R; y BENDERSKY, M (2003), Manual.gob. Estrategias de gobierno electrónico: la definición de un modelo de análisis y estudio de casos, Washington, BID.

VAN VEENSTRA A; KLIEVINK B; y JANSSEN M (2011), “Barriers and impediments to transformational government: Insights from literature and practice”, Electronic Government, an International Journal, vol. 8, num. 2, pp. 226-241. DOI: 10.1504/EG.2011.039838.

VÉLEZ-RIVERA B; RODRÍGUEZ-MARTÍNEZ M; DÍAZ W; NÚÑEZ-MOLINA M; y RIVERA-VEGA PI (2010), “Multidisciplinary e-government research and education as a catalyst for effective information technology transfer", en: Chen H-G (ed) Digital government: e-government research, case studies, and implementation, New York, Springer, pp. 670-695. DOI: 10.1007/978-0-387-71611-4_31.

YANG, T M; y MAXWELL, T A (2011), “Information-sharing in public organizations: A literature review of interpersonal, intra-organizational and inter-organizational success factors", Government Information Quarterly, vol. 28, pp. 164-175. DOI: 10.1016/j.giq.2010.06.008. 
YAO, Y; LEE, Y W.; HONG, P; y WENG, Z, (2010), "Power of Information Channels: Participation in e-Government Discourse”, AMCIS 2010 Proceedings, Lima, Paper 469.

YU, C-C; y JANSSEN, M (2010), “The need for strategic management and business model design in government and public administration”, Electronic Government, an International Journal , vol. 7, num. 4, pp. 299-315. DOI: 10.1504/EG.2010.035717

ZHAO, JJ; ZHAO, SY; y ZHAO, SY (2010), "Opportunities and threats: a security assessment of state e-government websites”, Government Information Quarterly, vol. 27, num. 1, pp. 49-56. DOI: 10.1016/j.giq.2009.07.004. 